\title{
Gefühlsräume - Raumgefühle Perspektiven auf die Verschränkung von emotionalen Praktiken und Topografien der Moderne
}

\author{
Benno Gammerl \\ Rainer Herrn
}

\begin{abstract}
Ausgehend von verschiedenen Ansätzen in der Forschung zu Gefühlen und Räumen umreißt der Artikel das theoretische, empirische und politische Potenzial des Spannungsfelds zwischen der affektiven Durchkreuzung geordneter Strukturen und der emotionalen Bearbeitung machtdurchwirkter Raumverhältnisse. Praktiken des urban gardening und Debatten über Gentrifizierung zeigen beispielhaft, wie Analysen der dynamischen Interaktionen zwischen Räumen und Gefühlen für eine kritische Perspektive auf Urbanität fruchtbar werden können. Die Beschäftigung mit der Körperlichkeit von Raumgefühlen und der Materialität von Gefühlsräumen bietet willkommene Gelegenheiten, nach Strategien zu suchen, die weder das beharrliche Wirken des Sozialen noch die widerständige Kraft des Materiellen in deterministischer Manier überbetonen, sondern sich stattdessen auf die ambivalenten Dynamiken und Effekte gefühlsräumlicher Prozesse konzentrieren.
\end{abstract}

Ersteinreichung: 8. November 2014; Veröffentlichung online: 9. August 2015

An english abstract can be found at the end of the document.

Gefühle spielen eine ausschlaggebende Rolle bei der gesellschaftlichen Herstellung von Räumen und bei der mitunter eigensinnigen Aneignung räumlicher Arrangements durch unterschiedliche Aktricen und Akteure. Diesen Zusammenhang versuchte in den letzten Jahren zum einen ein affekttheoretischer Ansatz zu erschließen, der den transgressiven, transhumanen und transsubjektiven Charakter affektiver Kräfte in den Vordergrund rückt (Thrift 2007, McCormack 2003). Dabei geraten heutige Städte zu „roiling maelstroms of affect“ (Thrift 2008: 171) und gleichsam zu Spielbällen von „inhuman and pre-subjective forces and intensities“ (Thrift 2008: 173).[1] Zum anderen entwickelte sich eine emotionsgeographische Perspektive, die sich weniger auf das unvorhersehbare und unregierbare Wirken der Affekte, als vielmehr auf sozio-kulturelle Ordnungen sowie auf subjektive Erfahrungen und Taktiken konzentriert, die den Blick auf bestimmte und bestimmbare gefühlsräumliche Muster lenken (Thien 2005: 450, Bondi 2005: 438). Ohne sich für eine der beiden Seiten dieses Gegensatzes zu entscheiden, der sich in der häufig anzutreffenden begrifflichen Differenzierung zwischen unvermitteltem Affekt und kulturell geformten Emotionen spiegelt, versucht unser Beitrag, das theoretische, empirische und politische Potenzial des Spannungsfelds zwischen der affektiven Durchkreuzung geordneter Strukturen und der emotionalen Bearbeitung machtdurchwirkter 
Raumverhältnisse für eine kritische Perspektive auf Urbanität fruchtbar zu machen.

Die emotionalen Dimensionen der Verräumlichung und der Unterwanderung von gesellschaftlichen Machtverhältnissen wurden bereits durch frühe Beiträge zur Stadtforschung berücksichtigt. Georg Simmel (1908: 645) ging davon aus, dass in der modernen Großstadt rationalinstrumentelle Beziehungen und eine blasierte Distanziertheit die, für ältere Siedlungsformen charakteristische, stärker emotional-intimen Bezüge zwischen den Bewohner_innen verdrängen. Auch die Vertreter der Chicago School streiften in ihrer Auseinandersetzung mit den Mechanismen sozialer Segregation die Frage der Emotionalität, indem sie die Bedeutung von Zusammengehörigkeits- und Rivalitätsgefühlen bei der Zergliederung des Stadtraums in attraktive und reizlose Zonen betonten (Park 1984: 5f.). Aus diesen Beobachtungen ergibt sich die nach wie vor relevante Einsicht, dass Emotionen ganz entscheidend zur Stabilisierung verräumlichter Machtverhältnisse beitragen können. Solche Großthesen schöpfen das Potenzial der Analyse des Zusammenhangs zwischen Räumen und Gefühlen jedoch bei weitem nicht aus. Können räumliche Arrangements hegemoniale Strukturen verfestigen, indem sie Angst einflößen oder Zuversicht wecken? Wann und unter welchen Umständen generieren dieselben Konstellationen stattdessen Emotionen wie Wut oder Enttäuschung, die zu einer Unterwanderung gesellschaftlicher Ordnungen beitragen können? Wie prägen umgekehrt Gefühlslagen die Wahrnehmung und die Nutzung machtvoller Räume? Uns geht es bei der Betrachtung der dynamischen Interaktionen zwischen Räumen und Gefühlen nicht darum, allgemeingültige Bezüge zwischen Raumformationen und Gefühlslagen zu postulieren. Im Zentrum stehen vielmehr kritische Perspektiven darauf, wie besondere gesellschaftliche Machtverhältnisse raum- oder ortsspezifische Gefühle prägen und wie sich umgekehrt Gefühle auf die soziale Produktion von Räumen auswirken. Die Konzentration auf Gefühle als teilweise unberechenbare und schwer bestimmbare Phänomene birgt ein produktives politisches und theoretisches Potenzial. Da sich emotionale Praktiken nicht auf die Reproduktion bestehender symbolischer und sozialer Ordnungen reduzieren lassen, rückt die Auseinandersetzung mit ihnen Möglichkeiten in den Blick, das Gegebene zu überschreiten und eröffnet zugleich methodische Auswege aus einer - im sozialkonstruktivistischen Sinn - unentrinnbaren, diskursiv hergestellten Wirklichkeit (Massumi 2002: 2, Kosofsky Sedgwick 2003: 6). Gleichzeitig ist uns jedoch auch nicht daran gelegen, in materialistischer Manier auf eine vorgängige Realität zu rekurrieren. Gerade die Beschäftigung mit der Körperlichkeit von Raumgefühlen und der Materialität von Gefühlsräumen bietet eine willkommene Gelegenheit, nach Strategien zu suchen, die weder das beharrliche Wirken des Sozialen noch die widerständige Kraft des Materiellen in deterministischer Manier überbetonen. Anstatt lediglich danach zu fragen, wie architektonische Gestaltungen die Gefühle von Aktricen und Akteure manipulieren oder wie Affekte Körper dazu bewegen können, bestehende Raumordnungen zu durchkreuzen, interessiert sich eine solche Perspektive vielmehr für gefühlsräumliche Prozesse, die weder unabhängig von sozio-kulturellen Strukturen ablaufen noch in diesen aufgehen. 


\section{Emotionale Kämpfe um städtische Räume: gärtnerische Beispiele}

Was damit gemeint ist, kann ein Blick auf emotionale Praktiken der Aneignung und der Kodierung von städtischen Räumen beispielhaft verdeutlichen. Dazu gehören Debatten über Gentrifizierung ebenso wie raumplanerische Unterscheidungen zwischen Arbeiten, Wohnen und Erholen oder bestimmte Formen touristischen Marketings. Wir möchten die Möglichkeiten und Schwierigkeiten einer gefühlsräumlichen Perspektive im Folgenden anhand neuerer Formen des Stadtgärtnerns veranschaulichen. Innerhalb dieses Feldes lassen sich verschiedene Taktiken alternativer Stadtraumbegrünungen wie das Guerilla-Gardening oder das Community-Gardening unterscheiden. Diese Bewegungen gingen zunächst von einer Kritik bestehender Raumordnungen aus, wobei sie nicht zuletzt deren emotionale Effekte im Visier hatten. Das gilt sowohl für die lebensreformerischen Ansätze der vorletzten Jahrhundertwende, die mit landesweiten Projekten wie der Schrebergartenbewegung auf die beschleunigte Verstädterung reagierten, als auch für die Strategie des Guerilla-Gardenings, die sich in den 1970er Jahren im Kontext verschiedener urbanitätskritischer Strömungen in den USA und in England entwickelte. Daran anknüpfend etablierten sich seither in vielen Ländern Praktiken zur Neuaneignung des Stadtraums, wie das Werfen von Samenbomben, das Bepflanzen von Baumscheiben, das Einrichten temporärer Gärten auf ungenutzten Brachen oder das Aufstellen von Pflanzkübeln, die die routinierten Nutzungen durcheinander bringen.[2]

Diese den Stadtraum verändernden Praktiken anarchistischer und umweltaktivistischer Gärtner_innen opponieren nicht zuletzt gegen die von ihnen als negativ wahrgenommen Stadtgefühle - die großstädtische Tristesse, die marktkonforme Langeweile oder die lethargische Gleichgültigkeit gegenüber dem als fremdbestimmt wahrgenommenen Umfeld (Gehrke 2012). Ihre Interventionen sollen demgegenüber ein authentisches Naturerleben, ein kreatives Sich-Betätigen im Freien oder ein selbstbestimmtes Gestalten des eigenen Umfeldes ermöglichen, um den „urbanen Lebensraum über wildes Gärtnern emotional zurückzuerobern"(Rademacher 2009).[3] Diese vielfältigen Motivationen verweisen auf ein emotionales Spektrum, das von Isolation, Entfremdung, Unzufriedenheit und Langeweile über Ärger und Empörung bis zu Hoffnung und Glücksgefühlen reicht. Stadtgärtnerische Projekte zielen häufig explizit auf emotionale Effekte ab. Sie wollen die „Gemeinschaft und das Zusammengehörigkeitsgefühl im Stadtviertel“ (Gehrke 2012: 4) stärken, ein „Gefühl für den Anbauprozess von Produkten“ (Allendorf/ Henney/Baake 2013) oder „das Gefühl für natürliche Produktionsprozesse“ (Gehrke 2012: 1) schaffen sowie das „verlorengegangene Gefühl ein Teil der Stadt zu sein“ wiederherstellen, um „wieder in Kontakt zu kommen mit der Natur und seiner [des Stadtmenschen] ursprünglichen Lebensweise“ (Gärtner München o. J.).

Allerdings bleibt eine gefühlsräumliche Analyse bei diesen positiven Selbstbeschreibungen der Aktricen und Akteure nicht stehen. Ohne das politische und emotionale Potenzial solch widerständiger Raumaneignungen zu bestreiten, konzentriert sie sich vielmehr auf die komplexe und nicht berechenbare Dynamik sowohl der intendierten räumlichen Veränderungen 
als auch des initiierten emotionalen Wandels. Die Begegnung mit ,Natur an ungewöhnlichen Orten kann nicht nur angenehme Reaktionen auslösen, sondern auch verstören oder behindern und daher auf Ablehnung stoßen. Gerade in der langen Frist - erinnert sei lediglich an den botanischen Umstand, dass sich eine Sonnenblume im Asphaltspalt nicht dauerhaft darauf beschränkt, herrlich gelb zu blühen - kann die Verschränkung räumlicher und emotionaler Dimensionen Effekte zeitigen, die den anfänglich intendierten Zielen diametral zuwiderlaufen. Das gilt ebenso für die häufig von urbanen Gartenprojekten bezweckte Intensivierung der Kommunikation innerhalb der heterogenen Bevölkerung. Auch die Berliner Initiative 10o\% Tempelhofer Feld begründete ihren erfolgreichen Volksentscheid über die „grüne“ Nachnutzung des ehemaligen Flughafengeländes nicht zuletzt mit dem Wunsch, einen „Raum der Begegnung aller sozialen Schichten und Kulturen“ zu schaffen und so „ein friedliches Miteinander“"zu ermöglichen.[4] Aber bleibt dieses Miteinander, dem man sich im Fall von intergenerationalen oder interkulturellen Kiezgärten nicht so ohne Weiteres entziehen kann, auch immer reibungsfrei? Zumindest die Debatte über das Tempelhofer Feld führte zunächst zu einem heftigen Konflikt, in dem sich - so könnte man argumentieren - die lokal-demokratische und emotional-intime Praxis der Initiative gegen die repräsentativ-demokratische und rational-instrumentelle Logik des Abgeordnetenhauses durchsetzte, das vor allem mit einem eher abstrakten Konzept von zunehmendem Wohnraumbedarf argumentierte.[5]

Am deutlichsten werden die ambivalenten gefühlsräumlichen Effekte alternativer Gärten dort, wo sie die Attraktivität ihrer Umgebungen erhöhen und Gentrifizierungstendenzen befördern, die die Initiator_innen und ersten Nutzer_innen verdrängen. Damit geht häufig die Legalisierung der zunächst ohne offizielle Genehmigung betriebenen Projekte einher, was wiederum das Maß behördlicher Steuerung und Reglementierung erhöht.[6] Durch diese neuen Formen der Anerkennung entfernt sich der gefühlsräumliche Charakter der Projekte immer weiter von der spontanen Strategie der gärtnerischen Guerillas, indem sie ihres subversiven Charakters beraubt zum bloßen dekorativen Accessoire einer Stadtraumverschönerung werden.

\section{Zwischen dem Universellen und dem Partikularen: ethnologische, psychologische, praxeologische und affekttheoretische Ansätze in der Gefühlsforschung}

Solche Überlegungen zur wechselseitigen Dynamik von Räumen und Gefühlen können auf eine breite theoretische Debatte zurückgreifen, die sich in den letzten Jahrzehnten entwickelt hat. Diese Forschungslandschaft prägt vor allem ein oft polemischer Gegensatz zwischen sozialkonstruktivistischen und universalistischen Ansätzen. Ethnologische und historische Arbeiten betonen meist, wie gesellschaftliche Strukturen und Muster das Fühlen bestimmter Gruppen von Aktricen und Akteuren prägen. Aus dieser Perspektive bestimmen beispielsweise die jeweils zur Verfügung stehenden Möglichkeiten des sprachlichen Ausdrucks von Gefühlen den Rahmen, innerhalb dessen sich die Empfindungen der Einzelnen bewegen (Abu-Lughod/ Lutz 1990, vgl. Rosenwein 2006). In ähnlicher Weise prägen auch das kulturspezifische Wissen über Emotionen oder bestimmte emotional relevante 
berufliche Routinen das Gefühlsleben individueller Aktricen und Akteure (Frevert et al. 2011, Hochschild 1979).

Universalistische Ansätze gehen dagegen davon aus, dass sich die Strukturen individuellen Fühlens transkulturell und transhistorisch grundlegend ähneln. Dieser Annahme folgt insbesondere der größte Teil der experimentellen psychologischen und neurowissenschaftlichen Forschung, die meist wenig an sozio-kulturellen Kontexten und Unterschieden interessiert sind, sondern sich eher auf beispielsweise durch Gehirnverletzungen verursachte Abweichungen vom sozusagen ,normalen emotionalen Verhalten und auf weltweit einheitliche Muster des mimischen Gefühlsausdrucks konzentrieren (Damasio 1994, Ekman/Rosenberg 2005). In ähnlicher Weise versuchen auch einige soziologische und historische Studien, universale Logiken des politischen Affekts oder kulturübergreifend gültige Grundregeln emotionaler Regime zu identifizieren (Ciompi/Endert 2011, Reddy 2001). Übertragen auf das Gartenbeispiel könnte man dementsprechend fragen, ob es eine anthropologische Konstante darstellt, dass Menschen Grünflächen als erholsam und anregend empfinden, oder ob sie erst im Verlauf der industriellen Moderne allmählich gelernt haben, Betongrau mit Stress und Langeweile, Grünpflanzen dagegen mit Entspannung und Kreativität zu assoziieren.

Zwischen diesen Polen oder jenseits von ihnen haben sich mittlerweile zwei weitere Herangehensweisen etabliert. Aus praxeologischer Perspektive resultieren emotionale Muster und Praktiken aus dem Zusammenspiel zwischen objektiven sozialen Strukturen und subjektiven Aneignungen oder Verkörperungen. Diese Ansätze verbinden die Annahme vergleichsweise stabiler habitueller Gefühlsmuster mit der Einsicht, dass deren performative Aufführung immer die Möglichkeit von Wandel und Abweichungen in sich birgt (Scheer 2012, Gammerl 2012). So rücken einerseits die emotionale Sozialisation oder das Erlernen gesellschaftlich adäquater Gefühlspraktiken und andererseits deren punktuelles Scheitern ins Zentrum des Interesses. Als beispielhaft für ein solches Scheitern wären etwa die möglichen Folgen eines Unterlaufens geschlechtsspezifischer Regeln für das Zeigen von Trauer zu nennen (Frevert et al. 2014, Shamir/Travis 2002). In einem ähnlichen Sinn ermöglicht auch die Guerilla-Begrünung neue emotionale Praktiken der Raumnutzung - sie verändert den Arbeitsweg und macht das brachliegende Nachbargrundstück zum Gemüsebeet -, gerade indem sie ältere soziale Praktiken wie die des Spaziergangs oder der Gartenarbeit aufgreift und neu interpretiert.

Affekttheoretische Zugänge betonen demgegenüber deutlich stärker das Moment der radikalen Überschreitung gesellschaftlicher Konventionen. Entgegen sozialkonstruktivistischen Positionen unterstreichen sie die unmittelbar körperlichen und außersprachlichen Dimensionen des Affekts. Mitunter gerät der Affekt dabei zu einem kritischer Reflektion nicht mehr zugänglichem Phänomen, das jeder Form des bewussten Denkens vorgelagert, in gewisser Weise übergeordnet ist (Thrift 2007, Conolly 2002).[7] Andere, in unseren Augen anschlussfähigere Positionen verorten den Affekt dagegen eher auf der Grenze zwischen Körper und Sprache oder im komplexen Feld der Beziehungen zwischen affektiver Virtualität und subjektiv erfahrener Aktualität. Dementsprechend ist der Affekt weder unabhängig von 
den jeweils gegebenen sprachlichen und politischen Strukturen, noch kann er auf diese reduziert werden. Daraus ergibt sich die Möglichkeit einer spezifisch affektiven Form der Kritik bestehender Verhältnisse (Berlant 2008, Gould 2010: 25-28, vgl. auch Massumi 2002, Kosofski Sedgwick 2003). Aus dieser Perspektive ist es nicht die radikale Alterität, sondern vielmehr die offene Vieldeutigkeit des Affekts, die - so das Argument - hegemoniale Muster der Intelligibilität unterläuft und kreatives Potenzial freisetzt, so dass beispielsweise die Überraschungseffekte von Samenbomben und von Blumen an Hauswänden eine radikale Verwirrung stiften können, die gänzlich neue gefühlsräumliche Konfigurationen entstehen lässt.

\section{Ausbaufähig: emotionale Dimensionen raumtheoretischer Debatten}

Auch raumtheoretische Ansätze können für eine relationale Betrachtung von Räumen und Gefühlen aufschlussreich sein, obwohl sie bisher kaum aus diesem Blickwinkel betrachtet wurden. Eine Diskussion dreier Klassiker des Genres - Henri Lefebvre, Michel Foucault und Michel de Certeau - kann das beispielhaft zeigen. In ihren Arbeiten beschäftigen sich diese Autoren entweder mit der gesellschaftlichen Produktion des Raums oder mit dessen vielfältigen Erschließungen durch eigenwillige Subjekte.[8] Henri Lefebvre überbrückt in gewisser Weise diesen Gegensatz, indem er den Raum als ein "gesellschaftliches Produkt [...], etwas durch die Produktionsverhältnisse und die darin eingebundenen Subjekte Hergestelltes“ (AnArchitektur 2002: 3) begreift. Dabei unterscheidet Lefebvre drei Ebenen, die auch aus gefühlsräumlicher Sicht von Interesse sind.

Der ,erfahrene oder wahrgenommene“ Raum (pratique spatiale) „,basiert auf einer nicht-reflexiven alltäglichen Praxis, die gesellschaftliche Verhältnisse als gegeben hinnimmt“ (AnArchitektur 2002: 17). Hier werden die der jeweiligen Gesellschaftsformation entsprechenden räumlichen Praktiken und die ihnen zugrunde liegenden Verhältnisse inklusive ihrer emotionalen Arrangements und Konnotationen produziert und reproduziert. Der ,erdachte oder abstrakt konzeptionierte' Raum (représentations de l'espace) konfiguriert sich in Form von Zeichnungen und Texten von Stadtforscher_ innen, Architekt_innen und Künstler_innen. Hier werden Raumgefühle zum Beispiel durch die architektonische Gestaltung von Vergnügungsparks vorgegeben, die auf die Evokation bestimmter Emotionen abzielt. Das wahrnehmende Subjekt wird auf diese Weise emotional gestimmt. Im ,gelebten Raum“ (espaces de représentation) werden „Bedeutungen [...] durch den Gebrauch hergestellt" und alternative Symbole und Imaginationen sowie minoritäre Erfahrungen, Traditionen und Erinnerungen können „vorherrschende Ordnungen und Diskurse unterlaufen“ (AnArchitektur 2002: 17). Es sind „Räume möglichen Widerstands und möglicher Kämpfe der Aneignung“, die „eine starke Motivation zu utopischen Veränderungen“ bergen (AnArchitektur 2002: 17). Diese Dimension ist für die Frage nach den Dynamiken und Ambivalenzen relevant, die sich aus Interferenzen und Reibungen zwischen den architektonisch-gestalterisch intendierten emotionalen Raumqualitäten und den unter Umständen widerständigen individuellen Raumaneignungen und -imaginationen ergeben können. 
Während der abstrakte Raum der Kontrolle vorrangig hegemonialen politischen und ökonomischen Interessen dient, entsteht der gesellschaftliche Raum aus alltäglichen Praktiken aller Gesellschaftsmitglieder, deren Materialisierungen die „erdachte[n] Grenzen und geregelte[n] Formen überschreite[n]“ (Gottdiener 2002: 23). In diesem Sinn opponieren die Protagonisten des Urban Gardening auch emotional gegen hegemoniale Raumkonzepte und verweigern sich der bloßen Reproduktion des wahrgenommenen oder erfahrenen Raums, indem sie beide im gelebten Raum durchkreuzen. Dass diese subversiven Durchkreuzungen wiederum auf alle drei Raumebenen - die in der Gleichzeitigkeit immer zusammenzudenken sind - zurückwirken, belegen etwa ihre gentrifizierenden Effekte.

Michel Foucault bereicherte die raumtheoretische Debatte mit seinem Begriff der „Heterotopie“, der nach den Funktionen sehr verschiedener Orte des Verworfenen fragt.[9] Dabei interessieren ihn besonders jene als „Gegenräume“ bezeichneten „lokalisierten Utopien“, „die sich allen anderen widersetzen und sie in gewisser Weise sogar auslöschen, ersetzen, neutralisieren oder reinigen sollen" (Foucault 2005: 10). Heterotopien werden von ihrer Funktion für die Gesellschaften her gedacht, ihre konkrete Gestaltung und stadträumliche Situierung folgt diesen funktionalen Zwecken. Dabei handelt es sich meist um institutionalisierte Arrangements, die bestimmten gestalterischen Logiken folgen. Foucault nennt den Garten als „das älteste Beispiel einer Heterotopie“ (Foucault 2005: 14-15) und beschreibt ihn - auch in seiner abstrahierten Version als Teppich - als einen idealen Sehnsuchtsort ohne jegliche Gefühlstrübung, Reibung oder Verwerfung. Obwohl Emotionen ansonsten im Konzept der Heterotopie kaum eine explizite Rolle spielen, bietet es dennoch verschiedene spannende Anknüpfpunkte für gefühls- und affekttheoretische Lesarten. Zum einen sind heterotope Orte wie der Friedhof, das Gefängnis, das Heim, das Obdachlosenasyl oder die Psychiatrie mit emotionalen Ausnahme- und Sonderzuständen, transgressiven Umbrüchen, biographischen Übergängen und unkontrollierten Affekten verknüpft und deswegen stark emotional aufgeladen. Gemeinsam ist ihnen die Überschreitung des Normativen. Zudem sind sie selbst oft mit spezifischen, gesellschaftlich problematischen emotionalen Registern verknüpft, wie der Friedhof mit der Trauer, das Gefängnis mit der Schuld, die Psychiatrie mit dem Wahnsinn sowie das Obdachlosenasyl mit Scheitern und Versagen. Zum anderen sind In- und Exklusionen emotionaler Lagen zentral für die Funktionsweise von Heterotopien, die dort entstehen, wo emotionale Verunsicherungen und/oder Verstörungen gleichsam in „andere Räume“ - so die gängige deutsche Übersetzung von „Heterotopien“ - ausgelagert werden. Als Gegenorte dienen sie somit der emotionalen Stabilisierung gesellschaftlicher Abläufe, indem sie instabile Gefühlslagen aus dem Alltag auslagern, was meist auf eine Stigmatisierung der Personage heterotoper Räume - Psychiatriepatient_innen, Gefangene, Fürsorgezöglinge, Alte, Tote etc. - hinausläuft. Aus gefühlsräumlicher Perspektive wird Foucaults Konzept deswegen vor allem dort interessant, wo sich aus dem Scheitern der klaren Trennung zwischen alltäglicher Routine und außeralltäglicher oder heterotoper Verunsicherung weitere Ambivalenzen und Dynamiken ergeben. So brachte die aus der antipsychiatrischen Bewegung resultierende Auflösung der psychiatrischen Anstalten und die Verlagerung ihrer Funktionen auf die 
Familienpflege oder auf Einrichtungen des betreuten Wohnens eine erneute, oft irritierende und Verunsicherung stiftende Sichtbarkeit psychischer Alterität in alltäglichen Begegnungen mit sich.

Michel de Certeau distanziert sich in seiner Kunst des Handelns (Certeau 1988) von Foucault, dem der bezüglich seines Verständnisses von Machtdispositiven vorwirft, dass er die Bedeutung subjektiver Handlungsspielräume verkenne. Deswegen betont de Certeau die Subjekte als eigensinnige und widerständige Aktricen und Akteure. Dementsprechend postuliert er zwei gegensätzliche Praktiken der Raumaneignung. Während der Voyeur den Raum aus einer starren und machtvollen Position von oben beobachtet, erschließen sich die einzelnen Aktricen und Akteure den Raum aus der Fußgängerperspektive von unten. Dabei entgehen de Certeaus Subjekte der Machtausübung im gelebten, im Gebrauch körperlich erfahrenen Raum, da dieser Raum dem distanzierenden Blick der Macht verborgen bleibt. Die Handlungen der Subjekte sind einzigartig, widersprüchlich und vielgestaltig und erweisen sich gegenüber vorgegebenen Konzepten und Gebrauchsweisen als resistent und subversiv (von Bismarck 2002). Wenn Räume erst durch Handlungen und in Behandlung entstehen, enthalten sie auch ein gestalterisches emotionales Potenzial. Als Beispiel können hier die Wegeplanungen in öffentlichen Grünanlagen dienen, deren vorgegebene Nutzungen oft ignoriert werden, wobei Trampelpfade entstehen, die von den Wegepräferenzen eigensinniger, widerständiger Benutzer_innen zeugen.

Aus gefühlsräumlicher Perspektive liegt die Stärke von de Certeaus Konzept gegenüber Foucault und Lefebvre in seiner Betonung der Flexibilität, ja nahezu Unabhängigkeit, und der Kreativität der subjektiven Raumpraktiken gegenüber den vorgegebenen Raumbedeutungen. Diese Betonung des Eigensinns und der Handlungsspielräume bietet viele Möglichkeiten für die Betrachtung der wechselseitigen Dynamiken von Raum und Gefühl. Allerdings geht de Certeau bei seiner Wiederermächtigung des handelnden Subjekts unter Umständen zu weit und betont vielleicht allzu einseitig dessen Widerständigkeit bei der Raumproduktion im alltäglichen Gebrauch. Denn obwohl sich aus dieser Perspektive gerade etwa die Transformation der negativen Stadtgefühle in subversive gestalterische Aktionen des Guerilla-Gardening schlüssig erklären ließe, sind bei weitem nicht alle diese Aktionen per se widerständig.

\section{Ambivalenzen und Dynamiken: die empirischen Potenziale von Gefühlsräumen und Raumgefühlen}

Ausgehend von den erwähnten emotions- und raumtheoretischen Ansätzen ergeben sich mitunter überraschende Korrespondenzen mit Überlegungen, die sich explizit mit den räumlichen Dimensionen des Emotionalen beschäftigen. William Reddys Konzept der „emotional refuges“ ähnelt beispielsweise Michel Foucaults Vorstellung von Heterotopien. Emotionale Fluchträume sind Nischen, innerhalb derer die strikten und eindeutigen Regeln eines emotionalen Regimes nicht gelten und die Einzelnen ihre gegen gesellschaftliche Konventionen verstoßenden Gefühle äußern und kultivieren können (Reddy 2001: 128f.).

Henri Lefebvres Beschreibung einer sozialen Produktion des Raums passt dagegen zu praxeologischen Ansätzen, dievon einer simultanen performativen 
Herstellung von Räumen und Gefühlen ausgehen (Reckwitz 2012). Dabei wirken räumliche und emotionale Dynamiken wechselseitig aufeinander ein, sodass spezifische materielle Assemblagen aus Dingen und Körpern besondere emotionale Praktiken ermöglichen, die sich wiederum auf die jeweilige Wahrnehmung der räumlichen Umgebung und damit auf deren weitere Ausgestaltung auswirken. In diesem Sinn zeigt Joseph Ben Prestels Beitrag in diesem Heft, wie durch die Neugestaltung der Friedrichstraße als Vergnügungsviertel um 1900 das respektable Flanieren und das despektierliche Sich-Prostituieren in eine prekäre Nähe zueinander gerieten. Diese Konstellation problematisiert die Frage, welche Mischung aus Amüsement, Angst und Schamhaftigkeit Frauen zur Schau stellen sollten, die als bürgerlich und anständig gelten wollten, obwohl sie abends allein in der Friedrichstraße verkehrten.[10]

Praxeologische Perspektiven erinnern mithin auch an Michel de Certeaus Überlegungen zum Gehen als individuelle Raumpraxis und -erfahrung. Noch deutlicher kommt de Certeaus Betonung der subversiven Eigenwilligkeit fußgängiger Subjekte jedoch in feministisch oder psychoanalytisch inspirierten emotionsgeographischen Arbeiten zum Tragen (Bondi 2005, Pile 2010). Diese Ansätze stellen die emotionale Aufladung bestimmter Orte oder Gegenden ins Zentrum und versuchen, subjektive Gefühlstopografien zu rekonstruieren. Dabei achten sie sowohl darauf, wie gesellschaftliche Strukturen und Machtverhältnisse persönliche Emotionslandschaften prägen, als auch darauf, wie die Aktricen und Akteure mit diesen Raumgefühlen jeweils umgehen. Diesen Fragen geht beispielsweise die kontrovers geführte Debatte über bestimmte Plätze und Situationen auf den Grund, die insbesondere für Frauen oder für Migrant_innen angstbesetzt sind (Valentine 1989, Pain 2009, Feministisches Kollektiv 2008). Auch Gabriele Dietze erkundet spezifische Emotionstopografien, indem sie untersucht, wie die expressionistische Künstler_innenbohème sich mittels alternativer emotionaler Zuschreibungen und Aufladungen im frühen 20. Jahrhundert die Berliner Kaffeehaus- und Kneipenlandschaft aneignete und dabei auch zu einer Verunsicherung bisher hegemonialer Vorstellungen von Männlichkeit beitrug.

Jenseits eines subjektzentrierten Erfahrungsbegriffs, wie er beispielsweise die Raumtheorien Lefebvres und de Certeaus prägt, fokussieren affekttheoretische Perspektiven die Rolle nicht-menschlicher Aktanten als gleichberechtigten Koproduzenten von Gefühlen, die mithin nicht im Inneren der Subjekte entstehen, sondern zwischen diesen und den Dingen, die sie umgeben. Aus dieser Perspektive erscheinen beispielsweise die heutzutage fast ubiquitären Bildschirme als zentral für die affektiven Funktionsweisen räumlicher Arrangements. Dabei unterstreichen affekttheoretische Analysen zumeist das politische Potenzial nicht-diskursiver und nicht-subjektiver Kräfte, die an den Rändern repräsentationaler Ordnungen Perspektiven auf unerwartete Dynamiken und Allianzen eröffnen. Damit wird der Affekt in gewisser Weise zum radikal Anderen der sozio-kulturellen Intelligibilität.[11] Katrin Köpperts Beitrag greift diese Ansätze in ihrer Untersuchung queerer Archive des ausgehenden 20. Jahrhunderts wie dem Schwulen Museum* in Berlin auf, indem sie das affektive Potenzial der älteren, teils ungeordneten und halb-privaten Sammlungsorte mit dem der neueren, offizieller wirkenden und häufig öffentlich finanzierten Archive kontrastiert. Zugleich stellt 
sie damit die spannende Frage nach den gefühlsräumlichen Bedingungen akademischer Wissensproduktion.

Paradoxerweise unterstreichen affekttheoretische Zugänge jedoch nicht nur die Unberechenbarkeit affektiver Ereignisse, sondern mitunter zugleich die medialen und technischen Möglichkeiten zur Manipulation emotionaler Räume oder zur ,creation of worlds', beispielsweise durch die glamouröse Inszenierung von Luxusprodukten (Thrift 2008, vgl. auch Chytry 2012).[12] Affekt wird aus dieser Perspektive zu einem System „of pipes and cables“, das die „basic mechanics and root textures of urban life“ trägt (Thrift 2007: 172). Diese technizistische Metaphorik wird vor allem von feministischer Seite kritisiert, weil sie das Subjekt letztlich zu einem Spielball exogener Kräfte macht (Thien 2005).

Diese Figur des Ausgeliefertseins greift in gewisser Weise phänomenologische Vorstellungen von räumlichen Gefühlen auf, die die Fühlenden gleichsam von außen überwältigen (Schmitz 1969). Für diese Ansätze ist das Konzept der Atmosphäre zentral, die das Fühlen der Einzelnen trägt, ohne dass diese sich dessen bewusst sein müssten (Böhme 2006). Damit ähneln solche Überlegungen wiederum älteren humangeographischen Annahmen über gleichsam universelle Raumgefühle, wie beispielsweise dem der Erhabenheit auf Berggipfeln oder dem der Angst in der Enge (Tuan 1974). Möglichkeiten, solche phänomenologischen Entwürfe produktiv weiterzudenken, diskutieren die Beiträge in der Rubrik Debatte, die sich mit Gaston Bachelards ,Poetik des Raums' auseinandersetzen.

Indem sie diese verschiedenen Ansätze und Dynamiken in den Blick nehmen, gehen die in diesem Heft vertretenen Analysen von Gefühlsräumen und Raumgefühlen deutlich über bisherige affekt- oder emotionsgeographische Perspektiven hinaus.[13] Neben diesem gemeinsamen theoretischen Anliegen verbindet die Aufsätze in diesem Heft zudem ein geographischer Fokus auf Berlin, der ihm eine reizvolle topografische Dichte verleiht. Gleichzeitig stellt die Betrachtung des frühen 20. wie des frühen 21. Jahrhunderts, eine gewisse historische Tiefe her. Indem es die diachrone Variabilität und die lokale Komplexität des Wechselspiels zwischen Räumen und Gefühlen andeutet - für die das Magazin weitere Belege aus unterschiedlichen Weltgegenden beisteuert -, möchte das Heft aktuelle Debatten innerhalb der kritischen Stadtforschung anstoßen und vorantreiben.

Ein weiteres, letztes Beispiel, das in einigen Einzelbeiträgen anklingt, soll das Potenzial dieser Perspektive verdeutlichen: Die Debatte über Gentrifizierungsprozesse gehört zum Kernrepertoire kritischer Stadtforschung. Allerdings beklagen einige Forscher_innen, dass sein inflationärer und häufig polemischer Gebrauch den Begriff allmählich aushöhle (Holm 2011). An diesem Punkt kann eine gefühlsräumliche Perspektive weiterhelfen, indem sie einerseits eine allzu ausschließliche Fokussierung auf ökonomische Fragen vermeidet und andererseits emotionale Ambivalenzen herausarbeitet, statt auf holzschnittartigen Oppositionen zwischen reichen Gentrifizierungstäter_innen und armen Gentrifizierungsopfern zu beharren.[14] Zwar spielen Rendite-Differenzen, Mieterhöhungen und Einkommensverteilungen zweifellos eine ausschlaggebende Rolle in Gentrifizierungsprozessen, aber ohne Verweis auf kulturelle Faktoren lassen sich die Verdrängungs- und Veränderungsprozesse nicht umfassend begreifen 
(Breckner 2010). Neben bestimmten, oft schichtspezifischen Vorstellungen von Urbanität und Modi des Umgangs mit kulturellen Differenzen prägen jedoch auch spezifische emotionale Dynamiken den als Gentrifizierung bezeichneten räumlichen Wandel. Dass diese bisher von der Forschung kaum berücksichtigt wurden, ist umso überraschender, als die öffentliche Debatte selbst häufig hoch emotional geführt wird.

Einige gefühlsräumliche Aspekte fügen sich relativ reibungslos in den gängigen Gegensatz zwischen eindringenden Neuankömmlingen und zurückweichenden Alteingesessenen. So verweisen Untersuchungen zu Sicherheitsarchitekturen beispielsweise auf das typische Muster einer asymmetrischen Verteilung von Angst auf diejenigen, die sie empfinden, und diejenigen, die sie auslösen (Low 2001). Ähnliches gilt für die Beschreibung bestimmter Wohngegenden als schmutzig und schambesetzt, während anderen Lagen eine ästhetisch genussreichere und kreativere Atmosphäre zugeschrieben wird, auf die die Bewohner_innen stolz sein sollen. Auch die Kontrastierung zwischen der Wut derjenigen, denen der Verlust ihrer Wohnung droht, und der mangelnden Empathie der Zuziehenden entspricht weitgehend der gewohnten Täter-Opfer-Gegenüberstellung.

Besonders spannend sind gefühlsräumliche Perspektiven auf Gentrifizierungsprozesse jedoch dann, wenn sie solche Oppositionen unterlaufen und verunsichern. In diesem Sinn ließe sich beispielsweise die Ökonomie der Ängste komplexer beschreiben, als es die simple Gegenüberstellung von Fürchtenden und Furchteinflößenden nahelegt. Einerseits müsste eine solche Analyse zwischen der spezifischen Räumlichkeit der Angst vor körperlicher und psychischer Gewalt und vor ökonomischer Deklassierung unterscheiden sowie andererseits intersektionale Überschneidungen zwischen verschiedenen Achsen gesellschaftlicher Marginalisierung berücksichtigen. Dort, wo - wie beispielsweise im westlichen Schöneberg - schwule Zuzügler auf eine ethnisch heterogene Bevölkerung treffen, mischen sich mitunter homophobe Töne in den Protest gegen die Gentrifizierung, während umgekehrt bestimmten ethnischen Gruppen in rassistischer Manier eine Unfähigkeit zur Toleranz sexueller Vielfalt unterstellt wird (Wolter 2012, vgl. auch Breckner 2010, Tsianos 2014). Eine Analyse, welche die auf allen Seiten involvierten gefühlsräumlichen Strategien berücksichtigt - auch diejenigen von Immobilienentwickler_innen, deren Hoffnungen und Vorstellungen durchaus nicht mit denjenigen der Kaufinteressent_innen kongruieren müssen -, könnte eine Reproduktion polemischer Gegensätze vermeiden und stattdessen ein vielschichtigeres Bild der Lage zeichnen.

Schließlich könnte man auch argumentieren, dass die ambivalente gefühlsräumliche Dynamik der Gentrifizierung dazu führt, dass die Hoffnungen aller Beteiligten letztlich enttäuscht werden. Während die Alteingesessenen meist nicht von den Transformationen profitieren können, die ein behaglicheres und angenehmeres Umfeld verheißen, lassen sich die Erwartungen der Neuankömmlinge ebenfalls nicht erfüllen, weil ihr eigenes Verhalten dazu führt, dass sich das attraktive gegenkulturell-authentische Milieu als bloßer Mythos entpuppt. Utopie und Nostalgie können auf diese Weise - wie eingangs bereits das Gartenbeispiel gezeigt hat - zu einem Konflikt zwischen unvereinbaren gefühlsräumlichen Entwürfen führen, der einem Stadtteil im Extremfall alle weiteren Entfaltungsmöglichkeiten versperrt. 
Obwohl die Beiträge dieses Heftes unterschiedliche Aspekte von Gentrifizierungsprozessen erörtern, reicht ihre thematische Vielfalt weit über dieses Feld hinaus. Angesicht dieser thematischen Vielfalt wird das Heft in erster Linie von drei gemeinsamen Fragen zusammengebunden: Erstens, in welchen Verhältnissen stehen räumliche und emotionale Dynamiken zueinander? Zweitens, wie lässt sich die Annahme, dass individuelle, situativ erzeugte Gefühlslagen die Wahrnehmung von Räumen bestimmen, mit der Einsicht verbinden, dass dieselben Räume zugleich die spezifische Subjektivität der Fühlenden strukturieren? Schließlich drittens, inwiefern kann der ,affektive Überschuss' von Raumgefühlen zu einer Verschiebung der Grenzen des Zeig-, Sag- und Machbaren und zu einer Verunsicherung der gesellschaftlichen Regeln der Bedeutungsproduktion beitragen?

\section{Endnoten}

[1] An dieser Stelle zitiert Thrift Spinks (2001: 24), vgl. auch McCormack (2003).

[2] In Berlin finden sich zahlreiche Beispiele für solche Einzel- und Gruppenaktionen (Prophet o. J.).

[3] Damit folgen diese Bewegungen einer Ausdehnung des Politischen durch neue affektive Register, die auch Thrift (2007: 182) beschreibt.

[4] Die Begründung der Initiative findet sich bei: wahlen-berlin.de, vgl. auch Berens (2010).

[5] So gesehen manifestierte sich in diesem Konflikt ein Gegensatz, der bereits Georg Simmel beschäftigte und den spätestens seit den 1990er Jahren auch Stadtplaner_innen intensiv diskutieren (Goodchild 1990).

[6] Vgl. dazu die minutiösen Vorschriften der Berliner Bezirksämter für die vormals wilde Baumscheibenbepflanzung, so beispielsweise des Bezirksamtes FriedrichshainKreuzberg (o. J.).

[7] Zur Kritik an diesen Ansätzen vgl. Leys (2011).

[8] Indem er insbesondere Henri Lefebvres und Michel Foucaults Überlegungen zum Raum einem englischsprachigen Publikum zugänglich machte, trug Edward Soja entscheidend zum sogenannten spatial oder topographical turn in den Geisteswissenschaften bei (Soja 1989, vgl. auch Döring 2010: 9of., Wagner 2010: 101).

[9] Wir beziehen uns in diesem Text auf die Übersetzung von Michael Bischoff(Foucault, 2005), die in Teilen wesentlich von der im Reclam-Band Aisthesis veröffentlichten Übersetzung von Walter Seitter (Foucault 1990) abweicht.

[10] Auch die emotionalen Effekte der nach dem 11.9.2001 eingerichteten Sicherheitsund Angstarchitekturen verdeutlichen die Wechselwirkungen zwischen räumlichen Strukturen und emotionalen Praktiken (vgl. Katz 2007).

[11] Thrift (2007: 175) beschreibt Affekt in paradoxer Weise zugleich als nicht-reflexiv und als eine Form des Denkens.

[12] Thrift (2007: 188) spricht in diesem Zusammenhang sogar von einer „new kind of velvet dictatorship“.

[13] Zur fehlenden Aufmerksamkeit der bisherigen Ansätze gegenüber der Variabilität und dem passageren Charakter emotionaler Muster und Praktiken vgl. Pain (2009: 471) und Kingsbury (2005).

[14] Zur Kritik an der Gegenüberstellung von Yuppies und Alternativen vgl. Lang (1998). 


\section{Autor_innen}

Benno Gammerl; Historiker, arbeitet u.a. zu imperialen Formationen, zur Geschichte der Gefühle und zur Zeitgeschichte der Homosexualitäten in Deutschland. gammerl@mpib-berlin.mpg.de

Rainer Herrn; Medizingeschichte, mit den Schwerpunkten Psychiatriegeschichte des 19. und 20. Jahrhunderts sowie Geschichte der Sexualwissenschaft und sexueller/geschlechtlicher Minderheiten.

Rainer.Herrn@charite.de

\section{Literatur}

Abu-Lughod, Lila / Lutz, Catherine A. (1990): Introduction: Emotion, discourse, and the politics of everyday life. In: Catherine A. Lutz / Lila Abu-Lughod (Hg.), Language and the Politics of Emotion. Cambridge u. a.: Cambridge University Press, 1-23.

Allendorf, Stefanie / Henney, Saskia / Baake, Sarah (2013): Urban Gardening. https:// selbstgemachtestadt.wordpress.com/2013/12/10/urban-gardening-2 (letzter Zugriff am 11.3.2015).

AnArchitektur e. V. (2002): Material zu: Henri Lefebvre: Die Produktion des Raums. Berlin: AnArchitektur.

Berens, Bettina [Landtag2010] (2010): Guerilla. Gärtnern für ein neues Wir-Gefühl. http:// landtag2010.blog.de/2010/04/o3/guerilla-gaertnern-neues-wir-gefuehl-8299926/ (letzter Zugriff am 28.10.2014).

Berlant, Lauren (2008): The Female Complaint: The Unfinished Business of Sentimentality in American Culture. Durham u. a.: Duke University Press.

Bezirksamt Friedrichshain-Kreuzberg von Berlin (o. J.): Baumscheibenbegrünung. http:// www.berlin.de/ba-friedrichshain-kreuzberg/politik-und-verwaltung/aemter/strassenund-gruenflaechenamt/gruenflaechen/artikel.146174.php (letzter Zugriff am 2.2.2015).

Bismarck, Beatrice von (2002): Hoffnungsträger - Foucault und de Certeau. In: Texte zur Kunst 12/47, 137-139.

Böhme, Gernot (2006): Architektur und Atmosphäre. Paderborn: Fink.

Bondi, Liz (2005): Making connections and thinking through emotions. Between geography and psychotherapy. In: Transactions of the Institute of British Geographers 30/4, 433-448.

Breckner, Ingrid (2010): Gentrifizierung im 21. Jahrhundert. In: Aus Politik und Zeitgeschichte 17, 27-32.

Certeau, Michel de (1988): Kunst des Handelns. Berlin: Merve Verlag.

Chytry, Josef (2012): Walt Disney and the creation of emotional environments: Interpreting Walt Disney's oeuvre from the Disney Studios to Disneyland, CalArts, and the experimental prototype community of tomorrow (EPCOT). In: Rethinking History 16/2, 259-278.

Ciompi, Luc / Endert, Elke (2011): Gefühle machen Geschichte. Die Wirkung kollektiver Emotionen - von Hitler bis Obama. Göttingen u. a.: Vandenhoeck und Ruprecht.

Conolly, William E. (2002): Neuropolitics: Thinking, Culture, Speed. Minneapolis u. a.: University of Minnesota Press.

Damasio, Antonio R. (1994): Descartes' Irrtum. Fühlen, Denken und das menschliche Gehirn. München: List.

Döring, Jörg (2010): Spatial Turn. In: Stephan Günzel (Hg.), Raum. Ein interdisziplinäres Handbuch. Stuttgart u. a.: Metzler, 90-99.

Ekman, Paul / Rosenberg, Erika L. (Hg.) (2005): What the Face Reveals: Basic and Applied Studies of Spontaneous Expression Using the Facial Action Coding System (FACS). Oxford u. a.: Oxford University Press, 2. Auflage.

Feministisches Kollektiv (Hg.) (2008): Street Harassment: Machtprozesse und Raumproduktionen, Wien: Mandelbaum.

Frevert, Ute / Scheer, Monique / Schmidt, Anne / Eitler, Pascal / Hitzer, Bettina / Verheyen, Nina / Gammerl, Benno / Bailey, Christian / Pernau, Margrit (2011): Gefühlswissen. Eine lexikalische Spurensuche in der Moderne. Frankfurt am Main u. a.: Campus. 
Frevert, Ute / Eitler, Pascal / Olsen, Stephanie / Jensen, Uffa / Pernau, Margrit / Brückenhaus, Daniel / Beljan, Magdalena / Gammerl, Benno / Laukötter, Anja / Hitzer, Bettina / Planper, Jan / Brauer, Juliane / Häberlen, Joachim C. (2014): Learning How to Feel. Children's Literature and Emotional Socialization, 1870-1970. New York u. a.: Oxford University Press.

Foucault, Michel (1990): Andere Räume. In: Karlheinz Barck / Peter Gente / Heidi Paris (Hg.), Aisthesis. Wahrnehmung heute oder Perspektiven einer anderen Ästhetik. Übersetzung von Walter Seitter. Leipzig: Reclam, 34-46.

Foucault, Michel (2005): Die Heterotopien. In: Michel Foucault, Die Heterotopien/Les hétérotopies, Der utopische Körper/Les corps utopique. Zwei Radiovorträge. Zweisprachige Ausgabe, übersetzt von Michael Bischoff, mit einem Nachwort von Daniel Defert. Frankfurt am Main: Suhrkamp, 7-22.

Gammerl, Benno (2012): Emotional Styles. Concepts and Challenges. In: Rethinking History $16 / 2,161-175$.

Gärtner München (o. J.): Urban Gardening in München. http://gaertnermuenchen.de/ urban-gardening-munchen (letzter Zugriff am 2.2.2015).

Gehrke, Janna (2012): Urban Gardening. Wie die Gärten in die Stadt zurückkehren. In: Impuls. Stadt - Land - Fläche 10. http://www.nabu.de/downloads/NABU-Impuls-StadtLandFlaeche/NABU-Impuls_SLF_102012.pdf (letzter Zugriff am 2.2.2015).

Gottdiener, Mark (2002): Ein Marx für unsere Zeit: Henri Lefebvre und die Produktion des Raumes. In: AnArchitektur e. V., Material zu: Henri Lefebvre, Die Produktion des Raums. Berlin: AnArchitektur, 22-26.

Goodchild, Barry (1990): Planning and the modern/postmodern debate. In: The Town Planning Review 61/2, 119-137.

Gould, Deborah (2010): On affect and protest, in: Janet Staiger / Ann Cvetkovich / Anna Reynolds (Hg.), Political Emotions. London u. a.: Routledge, 18-44.

Hochschild, Arlie Russell (1979): Emotion work, feeling rules, and social structure. In: American Journal of Sociology 85/, 551-575.

Holm, Andrej (2011): Ein ökosoziales Paradoxon - Stadtumbau und Gentrifizierung. In: Politische Ökologie 29/124, 45-52.

Katz, Cindi (2007): Banal terrorism. Spatial fetishism and everyday insecurity. In: Derek Gregory / Allan Pred (Hg.), Violent Geographies. Fear, Terror, and Political Violence. New York u. a.: Routledge, 349-361.

Kingsbury, Paul (2005): Jamaican tourism and the politics of enjoyment. In: Geoforum 36/1, 113-132.

Kosofsky Sedgwick, Eve (2003): Touching Feeling. Affect, Pedagogy, Performativity. Durham u. a.: Duke University Press.

Lang, Barbara (1998): Mythos Kreuzberg. Ethnographie eines Stadtteils 1961-1995. Frankfurt am Main u. a.: Campus.

Leys, Ruth (2011): The turn to affect: A critique. In: Critical Inquiry 37/3, 434-472.

Low, Setha M. (2001): The edge and the center: Gated communities and the discourse of urban fear. In: American Anthropologist 103/1, 45-58.

Massumi, Brian (2002): Parables for the Virtual. Movement, Affect, Sensation. Durham u. a.: Duke University Press.

McCormack, Derek P. (2003): An event of geographical ethics in spaces of affect. In: Transactions of the Institute of British Geographers 28/4, 488-507.

Pain, Rachel (2009): Globalized fear? Towards an emotional geopolitics. In: Progress in Human Geography 33/4, 466-486.

Park, Robert E. (1984 [1925]): The city. Suggestions for the investigation of human behavior in the urban environment. In: Robert E. Park / Ernest W. Burgess (Hg.), The City. Chicago u. a.: University of Chicago Press, 1-46.

Pile, Steve (2010): Emotions and affect in recent human geography. In: Transactions of the Institute of British Geographers 35/1, 5-20.

Prophet, Sandra (o. J.): Guerilla-Gardening, Bauerngärten, Schrebergärten. http://www. tip-berlin.de/kultur-und-freizeit/garntern-ohne-grenzen-guerilla-gardening-bauerngarten-schreibergarten (letzter Zugriff am 21.04.2015).

Reckwitz, Andreas (2012): Affective spaces: A praxeological outlook. In: Rethinking History 16/2, 241-258.

Reddy, William M. (2001): The Navigation of Feeling: A Framework for the History of Emotions. Cambridge u. a.: Cambridge University Press. 
Rosenwein, Barbara H. (2006): Emotional Communities in the Early Middle Ages. Ithaca u. a.: Cornell University Press.

Shamir, Milette / Travis, Jennifer (Hg.) (2002): Boys Don’t Cry? Rethinking Narratives of Masculinity and Emotion in the U.S. New York: Columbia University Press.

Scheer, Monique (2012): Are emotions a kind of practice (and is that what makes them have a history)? A Bourdieuan approach to understanding emotion. In: History and Theory 51/2, 193-220.

Schmitz, Hermann (1969): Der Gefühlsraum. Bonn: Bouvier.

Rademacher, Sylke (2009): Guerilla Gardening. http://blog.schoener-waers. de/2009/03/15/guerilla-gardening/\#more-187 (letzter Zugriff am 11.3.2015).

Simmel, Georg (1908): Soziologie. Untersuchungen über die Formen der Vergesellschaftung. Leipzig: Duncker \& Humblot.

Soja, Edward W. (1989): Postmodern Geographies. The Reassertion of Space in Critical Social Theory. London u. a.: Verso.

Spinks, Lee (2001): Thinking the post-human. Literature, affect and the politics of style. In: Textual Practice 15/1, 23-46.

Thien, Deborah (2005): After or beyond feeling? A consideration of affect and emotion in geography. In: Area 37/4, 450-456.

Thrift, Nigel (2007): Non-representational Theory. Space, Politics, Affect. London u. a.: Routledge.

Thrift, Nigel (2008): The material practices of glamour. In: Journal of Cultural Economy $1 / 1,9-23$.

Tsianos, Vassilis S. (2014): Homonationalismus und New Metropolitan Mainstream. Gentrifizierungsdynamiken zwischen sexuellen und postsäkularen Politiken der Zugehörigkeit, in: sub $\backslash$ urban 2/3, 59-80.

Tuan, Yi-Fu (1974): Topophilia: A Study of Environmental Perception, Attitudes, and Values. Englewood Cliffs: Prentice-Hall.

Valentine, Gill (1989): The Geography of Women's Fear. In: Area 21/4, 385-390.

Wagner, Kirsten (2010): Topographical Turn. In: Stephan Günzel (Hg.), Raum. Ein interdisziplinäres Handbuch. Stuttgart u. a.: Metzler, 100-109.

Wahlen-berlin.de (o. J.): Volksentscheid Tempelhofer Feld 2014. https://www.wahlenberlin.de/abstimmungen/VE2014_TFeld/allgeminfo.asp?sel1=6053\&sel2=1000 (letzter Zugriff am 8.11.2014).

Wolter, Salih Alexander (2012): Ist Krieg oder was? Queer Nation Building in BerlinSchöneberg, 15.6.2012, http://salihalexanderwolter.de/70 (letzter Zugriff am 10.4.2015).

\section{Spatial emotions - emotional spaces. Analyzing the entangle- ment of emotional practices and modern topographies}

Engaging with different approaches in research on emotions and spaces the article outlines the theoretical, empirical and political potential of the tension between affect as disrupting established structures and emotions as being shaped by given spatial conditions. Practices of urban gardening and debates about gentrification show by way of example, how analyzing the dynamic interplay between spaces and emotions can be rendered fruitful for critical vistas on urbanity. Considering the corporeality of spatial emotions and the materiality of emotional spaces enables a search for strategies that over-emphasize neither the persistent efficacy of the social nor the resistant force of the material in a deterministic fashion. Instead, it allows for bringing the ambiguous dynamics and effects of emotional-spatial processes into view. 
